\title{
Das Sprachlabor \\ und der \\ Fremdsprachenunterricht in Deutschland
}

\author{
by Udo O. H. Jung
}

Fehlinvestition Sprachlabor? Wie ein Fluch klebt dieses Schlagwort an mir, seit ich 1975 eine kleine Schrift unter diesem Titel herausbrachte.' Viele falsche Freunde haben mir seitdem anerkennend auf die Schulter geklopft: selbsternannte Sparkommissare in Ministerien und Behörden, aber auch einige ewig Gestrige und Menschheitsverbesserer, die Schüler entweder mit Kühen an Mlkmaschinen verwechselten oder sie in eine enthumanisierende technische Apparatur gepreBt sahen. So war's jedenfalls nicht gemeint! Häufig genug wurde das Fragezeichen einfach ignoriert. Aber selbst die, die sich die Mühe machten, das Buch näher anzusehen, hatten ihre Schwierigkeiten damit. Wie betont man das richtig? Indem man den monetăren Aspekt in den Vordergrund rückt und FEHLINVESTITION Sprachlabor sagt? Oder sollte es nicht vielmehr Fehlinvestition SPRACHLABOR heiBen, um anzeigen zu können, daB dieses Unterrichtsmittel nicht mehr so recht in die sprachdidaktische Landschaft passen will, vielleicht nie hineingepaBt hat?

Die Antwort aufdiese Frage können wir nur zu finden hoffen, indem wir noch einmal die Stationen des Sprachlabors an uns voribberziehen lassen, bevor wir uns den gegenwärtigen Problemen und den dazugehorigen Lösungsvorschlagen zuwenden. $\mathrm{DaB}$ wir dabei gleichzeitig unser Augenmerk auf die Entwicklung einer der wichtigsten Bezugswissenschaften der Fachdidaktik richten müssen - ich spreche von der Linguistik, genauer der Spracherwerbsforschung - ist selbstverständlich. Für die Retrospektive schlage ich die folgenden sechs Adjektive als Anhaltspunkte vor: 1. Restaurativ 2. Subversiv 3. Additiv 4. Integrativ 5. Kooperativ 6. ParaKommunikativ.

\section{Das Sprachlabor als Werkzeug der Restauration}

Erinnern wir uns. Als Ende der 50er, Anfang der 60er Jahre die Kunde vom Sprachlabor über den groBen Teich zu uns drang, konnte mit Fug und Recht die Frage gestellt und verneint werden, ob das Sprachlabor im deutschen Gymnasium eine Zukunft habe. ${ }^{2}$ Vier Jahre später bereits lautete die Gegenfrage, ob das deutsche Gymnasium ohne Sprachlabor eine Zukunft habe. ${ }^{3}$ Das Gymnasium war zu einem Hauptatbnehmer von Sprachlabors geworden und ist es bis zum heutigen Tag geblieben. Ein solcher Sinneswandel ist nur durch die Entwicklung zu erklären, die das deutsche Gymnasium in diesen Jahren durchgemacht hat. Georg Picht 
hatte inzwischen mahnend die Stimme erhoben und vor einer Bildungskatastrophe gewarnt. Studenten strömten auf die Dörfer, um die Landbevölkerung zu animieren, ihre Kinder auf die weiterführenden Schulen zu schicken. Beide wurden sie erhört, und das Gymnasium sah sich unversehens einem Schülerberg gegenüber, der in seiner Zusammensetzung alles andere als homogen war. Überfüllte Klassen und reduzierte Stundentafeln drohten, das Niveau des Gymnasiums unter die Grenze des Ertrăglichen absinken zu lassen. Warnend hieB es "Mag der Gedanke der Ausbildung einer Elite am Gymnasium auch zuweilen in den Hintergrund gedrängt werden, so enthebt das weder uns Lehrer noch die verantwortlichen Kulturpolitiker der Verpflichtung, dafür zu sorgen, daB die wirklich gut Begabten ohne Umwege zu hohen Leistungen geführt werden können. Hier werden sich letztlich Lebensfragen unseres ganzen Volkes im schulischen Alltag entscheiden." 4 Wie aber sollte es möglich sein, die Belange der gut Begabten zu berücksichtigen, die berechtigten Interessen der anderen nicht aus dn Augen zu verlieren und gleichzeitig das Niveau des deutschen Gymnasiums zu retten? Nun: "Die sich seit geraumer Zeit vollziehenden Umwandlungen in Gesellschaft und Arbeitswelt werden der Schule der Zukunft mit Sicherheit gröBere Verantwortung bei der Betreuung ihrer Schuler aufbürden, ohne daB sich das Reservoir qualifizierter Fachkräfte in gleichem $\mathrm{MaBe}$ erweitern wird. Deswegen sind schon heute Überlegungen am Platze, wie die Lehrkräfte von Routinearbeiten entlastet werden können. Eine MaBnahme zur Rationalisierung der Schülerbetreuung ist zweifellos der Einsatz von Sprachlaboratorien an Tagesheimschulen."s Stümperhafte Leistungen auf sprachpraktischem Gebiet waren auch schon im alten Gymnasium beobachtet worden, ${ }^{6}$ aber mit Hilfe des Sprachlabors sollte es nun gelingen, wenigstens diesen Stand zu halten, ihn möglicherweise gar zu verbessern. Dem Sprachlabòr wurden jene sperrigen Unterrichtsteile zugedacht, die die Nerven der Lehrer in besonderem MaBe belasteten; die Fachlehrer sollten freigesetzt werden für wichtigere Dinge, sollten sich wieder mehr den padagogischen Belangen Zuwenden können. Wenn darin auch eine emanzipatorische Note anklingt, die Hoffnungen, die man in das Sprachlabor setzte, waren in erster Linie restaurativer Natur. Das in Gefahr geratene Leistungsniveau der Schulen sollte wiederhergestellt werden.

\section{Das Sprachlabor als Mittel der Subversion}

Ein paar Jahre vor dem Sprachlabor hatte die strukturelle Linguistik Einzug in Deutschland gehalten. Sie vermittelte die letztlich selbstverstăndliche Einsicht, daB die gesprochene Sprache das eigentlich primäre Medium der Verständigung ist und fand bei all jenen ein bereitwilliges $\mathrm{Ohr}$, die ohnehin unzufrieden waren mit dem gängigen Fremdsprachenunterricht, dessen sprachpraktische Ergebnisse von Schubel ja als stümperhaft eingestuft worden waren. Gleichzeitig erschienen auf dem Markt erstmals amerikanische Methodiker, die die neuere Linguistik rezipiert und 
umgesetzs hatten und auch so gut mit den Ergebnissen der Verhaltensphychologie vertraut waren, daB sie das Sprachlabor als Kristallisationspunkt eines Neubeginns darstellen konnten. Das Sprachlabor galt als aparte Neuheit, dessen elektronisches Prestige dazu benutzt wurde, die Widerstănde gegen das neue Lernziel Sprechfertigkeit abbauen zu helfen. So diente das Sprachlabor in der beschriebenen Notsituation des Gymnasiums als Hebel, mit dem verkrustete Strukturen aufgebrochen werden konnten. Es erfüllte eine subversive Funktion.

\section{Das Sprachlabor wird additiv eingesetzt}

Dem Neuankömmling wurden zunächst Kellerrăume zugewiesen. Er war damit răumlich vom übrigen Unterrichtsgeschehen weit entfernt. Aber auch von der Sache her wurde die Trennung rasch offenbar. $\mathrm{Da}$ in den Schulstuben nach wie vor "Chalk and Talk" alten stils betrieben wurde, muBten die strukturellen Übungen im Sprachlabor als Fremdkörper erscheinen. Ihnen war die Aufgabe erteilt worden, die Schwächen des Klassenunterrichts auszubügeln. Und eine Zeit lang wetteiferten AA- und AAC-Labors darum, wer diese Aufgabe wohl besser erfüllen k8̈nne. Die Unterschungen sind Legion, die Ergebnisse aber äuBerst widersprüchlich.?

Erstals ein Kritiker auftrat, der das Sprachlabor in Bausch und Bogen zu verdammen entschlossen war, ${ }^{8}$ besann man sich darauf, daB mit der gemehrten Einführung von Sprachlabors die Ziele des Sprachunterrichts notwendig andere werden muBten und daB MeBinstrumente, die auf die alter Ziele geeicht waren, als untauglich auszuscheiden waren.

\section{Das Sprachlabor wird in den ubrigen Unterricht integriert}

Man könnte auch sagen, der Übrige Unterricht wird auf die Sprachlaborarbeit hin ausgerichtet. Inzwischen waren natmlich Lehrwerke - im Unterschied zu Lehrbüchern - konzipiert worden, die wie die Sprachlaborarbeit auf einer audiolingualen Basis standen und damit die Sprachlabortechnik, im Sinne von Vermittlungstechnik, in die Klassenzimmer transponierten. Damit war ein Weg beschritten, der Forderung, das Sprachlabor mUsse nicht nur inberall (nicht nur im Keller oder auf dem Dachboden), sondern auch jederzeit zur Verflgung stehen, Nachdruck zu verleihen.9 Konsequent wurde auch der zweite Weg beschritten. Visuelle und auditive Medien, wie Dia- und Overheadprojektoren, Tonbandgeråte und Kassettenrekorder wurden verstărkt in den Klassenunterricht einbezogen. Angestrebt wurde ein Verbund von Medien im Sprachunterricht, bei dem jedes, vom personalen Medium Lehrer bis zum audio-visuellen Lehrgerat gerade die Aufgaben erforlen sollte, für die es besonders geeignet war. Als unter solchen Bedingungen die Effektivitat des Sprachlabors bei einmal wöchentlichem Einsatz getestet wurde, zeigte sich, daB im Kontext eines Lehrwerks, das auch Tonbandubungen im Klassenraum vorsieht, das Sprachlabor keine meBbaren 
Leistungssteigerungen mit sich bringt.10 Vermittlungstechniken, nicht hardware ist ausschlaggebend. Die angestrebten Ziele lassen sich offenbar auch ohne Sprachlabor erreichen.

\section{Das Sprachlabor als Mittel der Kooperation}

Bei Einfthrung des Sprachlabors war zunatchst seine Individualisierungsfunktion stark in den Vordergrund gerffckt worden. Die Nathe zur Programmierten Instruktion wird allenthalben deutlich. Bangliche Schtler sollten im Sprachlabor ihre Sprechangste Uberwinden, langsame oder schnelle ihr Lerntempo nach Gusto einrichten konnen. Sehr bald jedoch wurde eine nicht zu unterschatzende Schwachstelle im vorherrschender 4-Phasen Drill ausgemacht. Gerade die Phase, die dem individuell Ubenden die Uberprufung seiner Leistung auf Korrektheit oder Irrtum hin ermø̈glichen sollte, erwies sich als relativ untaughlich. Bis zu $60 \%$ der Fehler blieben unentdeckt, weil die SchUler von der dritten, der Korrekturphase keinen effektiven Gebrauch machen konnten oder wollten." Ein Sprachlabor aber, das Fehler Systematisch ubt, ist unerwllnscht. Dies gilt unabhangig von der Frage, ob andere Unterrichtsformen entwickelt werden konnen, in denen dem Fehler eine notwendige Funktion im Sprachlernprozess eingeraumt wird. Dartuber wird spltter mehr zu sagen sein.

In dieser Situation muBte nach einer neuen Kontrollinstanz gesucht werden, die anders als der Lehrer nicht nur sporadisch präsent ist. Der Erkenntnis, daB die Schuller selbst diese Kontrollinstanz sein könnten, stand die schon beobachtete Unfahigheit der Lerner entgegen, ihre eigenen Fehler zu erkennen. Diese Beobachtung ist rein phânomenologisch betrachtet sicher nicht falsch. Ganz ohne Zweifel lassen die Schuller viele Fehler unkorrigiert zurtick. Nur gilt es, die als Ursache angenommene Unfthigkeit zu korrekter auditiver Diskrimination im H8̈rbereich zu befragen. Lange Zeit galt das Axiom, falsche Aussprache sei auf falsche oder mangelhafte Diskrimination zurefckzufthren, aus welchem Grunde auch diese Ubungsform an den Anfang jeglichen Sprachunterrichts gesetzt wurde. Es soll nicht bestritten werden, daB diie einwandfreie Diskrimination fremdsprachlicher Laute eine Voraussetzung zu einwandfreier fremdsprachlicher Produktion ist. Zweifelhalt ist und bleibt jedoch die Annahme, fehlerhafte Produktion sei ursåchlich auf fehlerhafte Dickrimination zurftckzufuhren oder anders gewendet, wer Fehler auf seinem Band zurücklasse, sei nicht in der Lage, Fehler als Fehler zu erkennen. Die beiden Fertigkeiten k8̈nnen durchaus auseinandertreten. ${ }^{12}$ So paradox es klingen mag, wenn man den Schuler, der fur seine eigene Produktion kein guter Richter ist, in eine Situation versetzt, in der er - unterstutzt auch durch visuelle Hilfen - zur Beurteilung der Produktion anderer herangezogen wird, laBt sich die Fehlerrate mit seiner Hilfe ganz erheblich drücken. Kooperatives Lernen tut dies. 
Technisch gesehen wird diese Arbeitsform durch einen einfachen Handgriff bewerkstelligt. Zwei nebeninander liegende Schülerkabinen werden dadurch miteinander verbunden, daB Schơler $A$ seine Mikrophon-Kopfhörer-Kombination (KMK) in die serienmafBig und zusatzlich eingebaute zweite Buchse von Schøler B steckt. Auf diese Weise behallt B die volle Verfügungsgewalt uber seine Maschine und leitet den Übungsfortschritt von A, d.h. er kontrolliert, zur Not mit Hilfe eines Antwortblattes den Lernfortschritt von $A$, hålt das Band bei Fehlern an, repetiert oder korrigiert fehlerhafte Produktion uber die Standleitung zwischen den beiden Kabinen. Auf diese Weise wird auch - durch den nun möglichen Ubergang vom 4-Phasen auf den 2-Phasen Drill - ein GroBteil der Monotonie aus der Sprachlaborarbeit genommen.'3 Aber Pattern Drill bleibt Pattern Drill. Die Grenze zum Pattern Drill kann nur Uberstiegen werden, wenn

\section{Das Sprachlabor mit Para-Kommunikativen Ubungen}

den Hiatus zwischen Strukturmusterobung und freier Konversation technologisch zu uberbrücken in der Lage ist. Para-kommunikative Ubungen basieren auf der vorhin skizzierten kooperativen Arbeitsweise. Wie der Name schon sagt, bleiben sie weitgehend im Vorfeld der eigentlichen Kommunikation, versuchen aber, die grøBtmôgliche Anrtherung an den Dialog, deren das Sprachlabor thig ist. Sie gehen davon aus, daB das Sprachlabor seine Aufgabe erst dann voll erftillt hat, wenn es sich selbst überflussig gemacht hat, wenn Schtiler und Mitschuler, Schuller und Lehrer oder gar Schüler und native speaker ungelenkt in der Fremdsprache miteinander kommunizieren. Viele Wege futhren zu diesem Ziel. Einer davon sei hier kurz skizziert. Als Beispiel nehmen wir Frank Stocktons bertihmte Geschichte "The Lady or the Tiger".14 Sie umfaBt ca. 2300 Worter und kann ohne Muthe auf mindestens ein Drittel ihres Umfangs reduziert werden. Stockton erzăhlt die Geschichte eines "halb-barbarischen K8̈nigs", der Gesetzesubertreter in einer speziell daftur gebauten Arena die Wahl zwischen zwei Tưren uberlät. Offnet dieser die eine, tritt eine Jungfrau hervor, mit der der Gesetzesbrecher auf der Stelle vermahlt wird. Seine Unschuld gilt als bewiesen. Hinter der anderen TUr verbirgt sich ein hungriger Tiger. Eines Tages wird ein Hofling beim Tete-a-Tete mit der KÖnigstochter erwischt und der nâmlichen Prozedur unterworfen. Die Prinzessin kann das Geheimnis der TÖren in Erfahrung bringen. Soll sie den Geliebten retten und damit an die Rivalin verlieren? Als der Jungling die Arena betritt, gibt sie ihm ein kaum merkliches Handzeichen. Ohne Zogern offfnet dieser die rechte Tưr. Hier bricht Stockton seine Erzathlung ab. Wir erfahren nicht, ob sich nicht doch die todbringende Bestie hinter dieser Tur verbarg. Aus diesem Basistext werden durch eine kleine Manipulation zwei grundlegend verschiedene Versionen. Wir fugen dem letzten Satz jeweils an: "Without the slightest hesitation, he went to the door on the right and opened it:
a( OUT CAME THE TIGER
b) OUT CAME THE LADY 
Auf diese Weise lassen sich zwei konträre Positionen aufbauen, die je nach Geschmack als unterschiedliche Versionen Stocktons, als Vorentwurf und Endversion oder auch wahrheitsgemăB als gezielte Manipulation deklariert werden können. Die Lerner werden nun in zwei Gruppen eingeteilt und bearbeiten einzeln oder innerhalb ihrer Gruppe kooperativ die ihnen zugedachte Version. Unter "Bearbeitung" k8̈nnen die unterschiedlichsten Arbeitsanweisungen subsumiert werden. So kann es ein Teilziel sein, tber eine Nachsprechubung nicht nur das inhaltliche Verstandnis zu sichern, sondern auch bestimmte lautsprachliche Erscheinungen wie Intonation, Vokalreduktion, Liaison mehr als sonst tblich zu trainieren. Bei alledem darf das Hauptlernziel nicht aus den Augen verloren werden. Es lautet: Den Schroler zu befahigen, in einem Streitgesprach die Wahrscheinlichkeit seiner Version gegentiber anderen $\mathrm{zu}$ verteidigen. Zu diesem Zweck wird man ihn vorab auf die zu erwartenden Schwierigkeiten einstellen und zwar inhaltlich wie sprachlich.

\section{A. Die gerichtete InhaltserschlieBung}

Die gerichtete InhaltserschlieBung verfolgt das Ziel, Handlungsweisen und Motive der Akteure im Hinblick auf den Ausgang der Geschichte zu gewichten. Im Falle der Version A muB die Liebe der Prinzessin als egoistische Opferung des Geliebten, im Falle B als heroische Hintansetzung ihrer Interessen gewertet werden.

Zu diesem Zweck werden Arbeitsblatter erstellt, die im Multiple-ChoiceVerfahren mogliche Antworten auf Fragen nach Handlungsgeschehen, Charaktereigenschaften der Personen etc. parat halten. Sie dienen als visuelle Stutzen fur die uber Tonband gestellten Fragen. Die Begrutndung, warum der jeweilige Nicht-Distraktor $\mathrm{zu}$ wăhlen ist, wird ebenfalls uber Tonband gegeben, so daB sich zwischen Tonband und Lerner ein erster, wenn auch gelenkter und von Entscheidungspausen unterbrochener Dialog abspielt. Naturlich sind unter den Distraktoren in Version $A$ ausgerechnet jene Argumente, die den Ausgang der Version B in besonderer Weise stûtzen, damit der spătere Diskutant fruthzeitig lernt, sich darauf einzustellen.

\section{B. Die sprachliche Vorbereitung}

Die sprachlichen Mittel, deren es sich zu bedienen gilt, um ein Gesprâch in Gang zu halten, und das heiBt in erster Linie, auf den Gesprâchspartner einzugehen, Prâsuppositionen in seiner Argumentatiion aufzudecken und $\mathrm{zu}$ benennen, Behauptungen $\mathrm{zu}$ falsifizieren, ihn zur Aufgabe seines Standpunkts zu bewegen oder schlicht Zeit zu gewinnen, vielleicht auch MiBverstândnisse aufzuklären oder Zugestâhdnisse zu machen, kőnnen nicht ohne weiteres vorausgesetzt werden. Soweit sie nicht im Kontaktunterricht bereits eingetibt wurden, muB die linguistische Bewältigung von Gespratchsstrategien nun systematisch geubt werden. Dies kann tber die Formuliierung von Fragen und Antworten im bereits erwathnten MultipleChoice-Test zur inhaltlichen ErschlieBung erfolgen; die relevanten Passagen 
Wăren besonders zu markieren. Einige Beispiele mőgen dies illustrieren.

1. Zur Anknïpfung an Vorausgegangenes

"A couple of minutes ago you agreed that...."

2. Zur Aufdeckung von Prăsuppositionen

"If that is so, how could she...."

3. Zuruckweisung von Behauptungen

"She can't have the pudding and eat it. In either case, it means...."

4. Uberredungstrategien

"It would be more in line with your previous

argument to say...."

5. Zeitgewinn

"Well, no, let me think. ..."

6. MiBverstândnisse aufklären

"I didn't mean it like that. ..."

7. Zugestandnisse machen

"I'd go along with you as far as...."

etc.

Am Ende dieser Ubungsphase sollte der Schøler nicht nur mit seiner Version bestens vertraut, sondern auch in der Lage sein, spielerisch für sie Partei zu ergreifen. Es wäre wohl aussichtslos, die Lerner nach Koppelung zweier Schülermaschinen aufzufordern, die Meriten ihrer jeweiligen Versionen aus dem Stand heraus zu diskutieren.

Der Ubergang muB von einem Tonbandmoderator Utbernommen werden, dessen Stimme auf dem Gerăt eines der Partner vorkopiert und festgehalten ist. Der Tonbandmoderator stellt abwechselnd Fragen an A und B, um allmthlich die Diskussion kontrovers zuzuspitzen, bevor er sich aus diesem Dreiecksverhaltnis zurtickzieht, um einen echten Dialog zwischen den Diskutanten entstehen zu lassen. Wie bei allen maschinell vermittelten Gesprächssituationen liegt hier der neuralgische Punkt. Der Dialog kann nach Ausscheiden des Moderators abrupt zusammenbrechen. Dem observierenden Lehrer bleibt jetzt nur noch die Option, mehrere Paare totber eine Konferenzschaltung zur erweiterten Gruppe zusammenzulegen. Er hat allerdings im vorhinein bereits durch die Auswahl der Partner eine gewisse Vorsorge getroffen, damit diese letzte Phase forcierter Kooperation moglichst lange hiinausgezogert werden kann.

Őffensichtlich ist die Programmerstellung ein sehr aufwendiger Prozess, viel aufwendiger als bei den gângigen Pattern Drills, seien Sie nun anspruchslos manipulativ oder situativ eingebettet. Und natürlich stellt sich die Frage, ob es sich bei para-kommunikativen Ubungen nicht bloB um einen Trick handelt, mit dem das Sprachlaborsterben nur verżogert, nicht aber verhindert werden kann. 
Ist nicht vielleicht das Sprachlabor ein in Elektronik sich materialisierendes falsches Konzept von der Art und Weise, wie man eine zweite oder fremde Sprache erwirbt? Eine so teure Investition vorzunehmen, kann doch nur dann sinnvoll sein, wenn nachweisbar ist, daB diese Apparatur die ohnehin notwendige Arbeit des Lehrers unterstlitzt, sie verkllrzt und optimiert.

An der dominierenden Rolle des Lehrers als oberster Steuerungsinstanz im Fremdsprachenunterricht sind nun allerdings in letzter Zeit ganz erhebliche Zweifel aufgekommen. Wer eine kybernetische Schltusselstellung einnehmen will, muB wissen, an welcher Stelle seine Steuerungsbemothungen sinnvoll ansetzen kơnnen. Die Fremdsprachendidaktik hat jedoch einem altbekannten Glied im Dreiecksverhältnis von Stoff - Lehrer Schuler eine ganz neue Dimension abgewinnen k8̈nnen, durch die bisherige Praktiken in Frage gestellt werden.

\section{Natefrlicher Zweitsprachenerwerb und Fremdsprachenunterricht}

Dab Menschen thig sind, Sprache zu erwerben, wird auf eine angeborene Disposition zurtackgefthrt. Diese Fathigkeit, aus den primåren sprachlichen Daten der Umgebund eine Grammatik der Sprache aufzubauen und so Ober ein begrenztes Regelinventar eine unendliche Zahl korrekter ÄuBerungen zu erzeugn, macht das Kind dem Linguisten vergleichbar. Letzterer versucht, den Erwerbsprozess des Kindes zu verfolgen. Er beschreibt ihn als eine geregelte Abfolge von Stadien, der kein Spracherwerber entgehen kann, die keine noch so groBen Bemuhungen erwachsener 'Lehrer' umkehren können, deren Durchlaufzeit bestenfalls verktitzt werden kann.

Ein solcher streng hierarchisch organisierter ProzeB wiederholt sich, beim naturlichen Zweitsprachenerwerb. Damit ist ein Spracherwerbsprozess gemeint, der eintritt, wenn eir. Sprecher seine Muttersprache bereits vollstandiig oder weitgehend erworben hat, pløtzlich in eine fremdsprachige Umgebung versetzt wird und nun ohne die Hilfe formeller Unterrichtsverfahren auch die neue Sprache erwerben muB. Beispiele sind Gastarbeiter-, Diplomaten und Soldatenkinder. Aus der RegelmäBigkeit der dabei ablaufenden Erwerbsprozesse muB geschlossen werden, daB derselbe Mechanismus wie beim Erstsprachenerwerb oder ein vergleichbarer Mechanismus aktiviert wird. Er reagiert - wie beim ersten Mal - auf die sprachlichen Daten der Umgebung. Ich werde den Vorgang am Beispiel des Erwerbs der Negation bei Englisch als Zweitsprache durch deutsche Lerner erläutern. Ich greife dabei auf die Ergebnisse der Kieler Schule zurffck.'15

Bis zur normgerechten Ausbildung der linguistischen Mittel, mit deren Hilfe im Englischen negiert wird, durchlaufen die Kinder verschiedene Spracherwerbsstadien. Sie beginnen mit der holophrastischen Negation. Sie ist gekennzeichnet durch die Verwendung des schlichten "No", dem keine weiteren Konstituenten vorausgehen oder nachfolgen. Sobald in 
der Mehrwortnegation weitere Konstituenten zum Negationsmorphem hinzutreten, muB zwischen anaphorischer und nicht-anaphorischer Negation unterschieden werden.

Wie der Name andeuten will, bezieht sich die Negationspartikel bei anaphorischer Negation nicht auf Konstituenten derselben ÄuBerung, sondern auf Vorausgegangenes, selbst wenn dieses nicht explizit gemacht wird. Eine RuBerung wie

\section{(1) no, Mommy}

will soviel bedeuten wie: "Nein, Mammi, (nicht der Papi) soll das machen." Bei nicht-anaphorischer Negation verhält es sich umgekehrt:

(2) no finish

kann soviel bedeuten wie: "Ich bin noch nicht fertig. Verneint wird die mit "no" zusammen auftretende Konstituente.

Als nachstes werden satzinterne Negationen registriert. "No" steht also nicht mehr am Anfang (oder auch Ende) des Satzes, sondern wird von anderen Satzgliedern eingeschlossen:

\section{(3) lunch is no ready}

Innerhalb dieses Stadiums ist eine Subklassifikation nach Satztypen m8glich. Zunächst werden Åquationalsåtze, d.h. Sätze, in denen die Kopula das verbale Element darstellt, satzintern negiert, Vollverbsatze treten erst spatter hinzu. "No" und "not" variieren zu diesem Zeitpunkt noch frei. Nachdem sich die Verwendung von "not" einigermaBen stabilisiert hat, treten auch die ersten Belege fur do-Periphrase auf. Dies geht jedoch miit einer Übergeneralisierung einher. Die Kinder machen keinen Unterschied zwischen Vollverben und Hilfsverben.

\section{(4) I don't can eat anymore}

Dies müge genưgen, un $\mathrm{zu}$ veranschaulichen daB auch der natürliche Zweitsprachenerwerb eine geordnete Abfolge von Spracherwerbsstadien darstellt.

Welche Bedeutung hat nun diese Beobachtung aus dem naturlichen Fremdsprachenerwerb ftt den Fremdsprachenunterricht? Es bedarf keiner Frage, daB sich die beiden Situationen grundlegend unterscheiden. Im ersten Fall befindet sich der Lerner unter andauernder, aber unsystematischer fremdsprachlicher "Berieselung", im zweiten bemthen sich speziell ausgebildete Lehrkrafte stundenweise, die zu lernenden Daten in einer geordneten Folge vom Leichten zum Schweren behutsam an ihn heranzutragen. Ist dort eine unmittelbare Motivation durch den Wunsch zur Teilhabe am Gemeinschaftsleben gegeben, so muB sie hier mothsam ther lange Zeitråume hin künstlich aufrecht erhalten werden. Daran ist nicht zu zweifeln, und darum geht es auch nicht. Die Frage lautet, ob jener beim naturlichen Erstsprachenerwerb ebenso wie 
beim natUrlichen Zweitsprachenerwerb tiber die geregelte Abfolge der Spracherwerbsstadien in seiner Tatigkeit beobachtbare Spracherwerbsmechanismus im Fremdsprachenunterricht plotzlich vom Lerner suspendiert wird, weil die Prassentation der fremdsprachigen Daten nicht mehr ungeordnet erfolgt oder ob er nicht doch angesichts fremdsprachiger Daten unwillkurlich zur Entfaltung kommt, obwohl die Lehrstrategie so tut, als stande ihr jener Spracherwerbsmechanismus als voll manipulierbares Element im Lernprozess zur Verfugung. Anders formuliert: Die Fremdsprachendidaktik hat bisher so getan, als ob dieser Spracherwerbsmechanismus eine bloBe Rahmenbedingung sei, eine leere Disposition Sprachen zu lernen, deren konkrete Aufferlung in ihr Belieben gestellt sei. Wenn dem aber nicht so ist, wenn der Spracherwerbsmechanismus nicht unterdritckbar ist und angesichts sprachlicher Daten zu eigengesetzlicher Entfaltung kommen will, dann ware es straflich, im Fremdsprachenunterricht an dieser vom Schuller in den LernprozeB eingebrachten Vorbedingung vorbeizugehen. Schlimmer noch, der Lehrer müte immer dann, wenn der vom Schưler intendierte Weg zu seinen Lehrstrategien verguer lauft, einen Unterdrttckungskampf fohren. Und wenn man sich die derzeitigen Ergebnisse des Fremdsprachenunterrichts ansieht und die im naturlichen Zweitsprachenerwerb erzielten tberdurchschnittlich guten und schnellen Erfolge dagegen hâlt, kommt man zu dem nicht abwegigen SchluB, daB das schlechte Abschneiden des institutionellen Fremdsprachenunterrichts die Folge eines solchen Kampfes sein kơnnte.

Es ware sicherlich falsch, wenn der Fremdsprachenlehrer eine solche Schelte allein auf seine Person ziehen wïrde. DaB er z.Zt. nicht in der Lage ist anzusagen, bei welchem Substrat seine Steuerungsbemothungen anzusetzen hatten, ist schlicht eine Folge des Forschungsdefizits in der Spracherwerbsforschung, respektive der Tatsache, daB die Fachdidaktik sich unter den ihr vorgegebenen Bedingungen bisher nicht als eigenstandiger Wissenschaftszweig etablieren konnte. Dennoch bedeutet dies bezogen auf das Sprachlabor - daB wir zwar tober ein technisches Instrumentarium verfugen, welches in der Lage ist, bestimmte Funktionen des Lehrers zu objektivieren, zu vervielfaltigen, ja zu optimieren, daB wir aber keinen wirklich sinnvollen Gebrauch davon machen konnen, weil wir nicht einmal wissen, an welcher Stelle und in welcher Weise der zu ersetzende oder zu stutzende Lehrer im LernprozeB optimal auf den Plan zu treten hat. Oder und um ein solches Diktum abzumildern, wir kÜnen im Vorgriff auf zuktmftige Ergebnisse der Spracherwerbsforschung und der daran anzuschlieBenden fachdidaktischen Forschung nur versuchen, Ubungstypen im Sprachlabor anzusetzen, die nur in ganz globaler Weise steuern. Parakommunikative Ubungen gehoren zu diesem Typ. Eine noch radikalere Lơsung besteht im Einsatz des Sprachlabors als Mittel zur Prasentation vollig unstrukturierten Materials, um so nahe wie moglich an eine naturliche Erwerbssituation heranzukommen. ${ }^{18}$ 


\section{Anmerkungen}

1 U. Jung \& M. H. Haase (Hgg.), Fehlinvestition Sprachlabor? Beiträge zu einem konstruktiven Sprachunterricht mit technischen Medien, Kiel, 1975. Demnáchst in uberarbeiteter und teilweise erweiterter Fassung als U.O.H. Jung (Hg.), Das Sprachlabor. MOglichkeiten und Grenzen technischer Medien im Unterricht, Kronberg/Ts., 1978.

2 R. Freudenstein, "Hat das Sprachlabor eine Zukunft im deutschen Gymnasium?" Die Neueren Sprachen 6/1961, S. 284-286.

3 R. Freudenstein, "Hat das deutsche Gymnasium ohne Sprachlabor eine Zukunft? Erkenntnisse nach einem Arbeitsversuch", Das Sprachlabor und der audiovisuelle Unterricht, 1/1965, S. 4-11.

4 A. Bemmerlein, "Das Sprachlabor am Gymnasium - Uberlegungen und Erfahrungen nach zweijthriger Praxis im franzosischen Elementarunterricht", Das Sprachlabor in der Unterrichtspraxis, hg. Anton Bemmerlein, Freiburg im Breisgau, 1967, S. 66 f.

5 A. Bemmerlein, a.a.O., S. 61. Die Hervorhebungen stammen von mir.

6 F. Schubel, Methodik des Englischunterrichts für hôhere Schulen, Frankfurt a.M., 19664, S. 20.

7 R. F. Keating, A Study of the Effectiveness of Language Laboratories, Institute of Administratiive Research, Teachers College, Columbia University, New York, 1963. Scherer, G.A.C. \& Wertheimer, M., A Psycholinguistic Experiment in Foreign Language Teaching, New York, 1964. Ph.D. Smith, A Comparison of the Cognitive and Audiolingual

Approaches to Foreign Language Instruction, Philadelphia, 1970.

L. Levin, comparative studies in foreign-language teaching, Stockholm, 1972.

R. Olechowski, Das Sprachlabor. Theorie - Methode - Effektivitat, Wien, 1973.

8 M. Mumme, "Sprachlabore oder Aporien der Verwertungsinstruktion", Sprache im technischen Zeitalter, 48, 1973, S. 285-308.

9 R. Freudenstein, "Zehn Jahre Sprachlaborarbeit - REckblick, Stand und Ausblick", Programmiertes Lernen Unterrichtstechnologie und Unterrichtsforschung, 8, 1971, 2, S. 86-92.

10 P.S. Green (Hg.), The Language Laboratory in School. Performance and Prediction. An Account of the York Study, Edinburgh, 1975.

11 R. Lademann, "Zu Fragen der Ausstattung von Sprachlabors fur allgemeinblidende schulen," Erfahrungsberichte aus der Sprachlaborarbeit, Beiheft 2 zu pl, Berlin, S. 36-41. 
G. Desselmann, Ubungsgestaltung im Sprachlabor. Zur Theorie und Praxis des Deutschunterrichts fur Auslander, Leipzig, 1972².

$12 \mathrm{Vgl}$. W. Nemser, "The predictability of interference phenomena in the English speech of native speakers of Hungarian", Papers in Contrastive Linguistics, hg. G. Nickel, Cambridge, 1971, S. 89-96.

13 Vgl. U. Jung, "Co-operative Learning in the Language Laboratory", System, 3/1974, S. 21-26.

14 Huebers Fremdsprachliche Texte, Nr. 2, München 19694.

15 Vgl. H. Wode, "Naturliche Zweitsprachigkeit: Probleme, Aufgaben, Perspektiven," Linguistische Berichte, 32, 1974, S. 15-36.

S. Felix, Linguistische Untersuchungen zum englisch-deutschen Zweitsprachenerwerb unter natürlichen Bedingungen, Habilitationsschrift, Kiel, 1976. Demnåchst als Buchpublikation, München: Fink, 1978.

16 S. Felix, "Language Acquisition and Language Learning", erscheint in System, $2 / 78$.

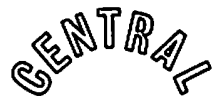

CONFERENCE ON THE TEACHING OF FOREICN LANGUAGES

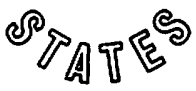

FOREIGN LANGUAGE INSTRUCTION IN THE 1980's

ALTERNATIVES, BASICS, COMPETENCIES

MEETING IN COOPERATION WITH

MINNESOTA COUNCIL ON THE TEACHING OF FOREIGN LANGUAGES

DATE: April 19-21, 1979 (as always, the weekend after Easter)

PLACE: Minneapolis, Radisson South and L'hotel de France (formerly Sofitel)

HIGHLIGHTS:

- Keynote Addresses

- Thursday Workshops

- Special Interest Sessions

- Language Sessions

- Luncheon, Banquet and Entertainment

THURSDAY EVENING SPECIAL: Wine and Cheese Fote in exhibit area

Companies or organizations wishing to exhibit, anyone seeking Advisory Council membership, and persons interested in receiving Conference Program Booklet containing registration and hotel reservation forms should write to: Maurice W. Conner, Department of Foreign Languages, University of Nebraska at Omaha, Omaha, Nebraska 68101. (402) $554-2403$ 